\title{
Microarray analysis of long non-coding RNAs related to microRNA-148b in gastric cancer
}

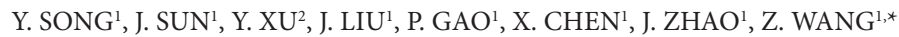 \\ ${ }^{1}$ Department of Surgical Oncology and General Surgery, ${ }^{2}$ Department of Breast Surgery, First Hospital of China Medical University, 155 North \\ Nanjing Street, Heping District, Shenyang, Liaoning, 110001, P.R. China \\ *Correspondence: josieon826@sina.cn
}

Received July 7, 2016 / Accepted October 10, 2016

\begin{abstract}
The miR-148/152 family (miR-148a, miR-148b, miR-152) is differentially expressed in gastric cancer tissues and nontumor tissues. Our previous studies indicated that miR-148/152 family is important in the tumorigenesis and development of gastric cancer. We also found several target genes that were regulated by the miR-148/152 family and several factors that could influence members of this family. However, the molecular mechanisms and function of this family in gastric cancer remain unclear. Recently, long non-coding RNAs (lncRNAs) have been found to play an important role in the pathogenesis of many diseases. The aim of the present study was to perform expression profiling to identify lncRNAs that might be associated with miR-148b in gastric cancer and predict their potential functions. Total RNA was extracted from gastric cancer cell line SGC-7901 treated with miR-148b mimics and from untreated gastric cancer cells. Microarray analysis was performed using the Agilent LncRNA + mRNA Human Gene Expression Microarray V3.0 platform, which was designed for the profiling of human lncRNAs and protein-coding transcripts. A total of $37581 \mathrm{lncRNA}$ and $34303 \mathrm{mRNA}$ transcripts were detected in gastric cancer cell line SGC-7901 and cell line SGC-7901 treated with miR-148b mimics. Among the differentially expressed lncRNAs, 240 up-regulated and 64 down-regulated lncRNAs were identified. XLOC_000983 was the most up-regulated lncRNA, and M18204.1 was the most down-regulated lncRNA. Among the differentially expressed mRNAs in different cell lines, 196 were consistently up-regulated and 320 were consistently down-regulated. POSTN was the most up-regulated mRNA, and HBB was the most down-regulated mRNA. Gene Ontology (GO) and pathway analyses indicated that the lncRNAs influenced by miR-148b might play an important role in the immune system and were associated with the development of gastric cancer. The altered expression of lncRNAs influenced by miR-148b may play a partial role in pathways implicated in gastric cancer development and progression, such as the immune response pathway.
\end{abstract}

Key words: long non-coding RNA, microRNA, microarray, gastric cancer, immune system

Gastric cancer affects approximately one million people per year; it is one of the most commonly diagnosed cancers and the second most frequent cause of cancer-related mortality worldwide [1]. A large number of gastric cancer patients are diagnosed at an advanced stage, and the 5-year survival rate of gastric cancer is less than 25\% [2]. Despite the improvements in radiotherapy, chemotherapy, targeted therapy, and surgical techniques in the past few years, the survival rate for gastric cancer patients remains low [3]. One reason for the low survival rate is the lack of specific molecular biomarkers for early diagnosis. Furthermore, only a few target sites are effective for treatment of gastric cancer, and only a few targeted drugs, such as Trastuzumab and Apatinib, are effective for some late-stage gastric cancer patients $[4,5]$. Therefore, suitable predictive biomarkers are urgently needed for the treatment of gastric cancer patients.

In recent years, noncoding RNAs (ncRNAs) have been considered as key regulators; theses RNAs participate in various biological processes and provide a novel view of the traditional central dogma of molecular biology [6]. NcRNAs can be grouped into short ncRNAs and long ncRNAs (lncRNAs) according to their transcript sizes [7]. MicroRNAs are the most relevant short ncRNAs, considering that they play a major role in the regulation of various cellular processes, including cellular differentiation, proliferation, and apoptosis by repressing the expression of their target genes. Of note, a large number of miRNAs have been shown to be involved in cancer and may regulate the processes of 
tumorigenesis, proliferation, invasion, and metastasis. For example, miR-122 has been reported to be dysregulated in many types of cancers, such as gastric cancer, colorectal cancer, and hepatocellular carcinoma [8-10]. Moreover, miR-122 has been used as a target therapy site in chronic hepatitis $\mathrm{C}$ virus infection and has provided a novel strategy for the control of hepatocellular carcinoma [11]. However, the mechanisms involved in the regulation of miRNAs in the complex network of cancer development are poorly understood.

MiR-148a, miR-148b and miR-152 are members of the miR-148/152 family and have the same seed sequence, with approximately 6-7 nucleotides [12]. These members play distinct roles in many types of tissues, including tumor, non-tumor, and normal tissues. The miR-148/152 family is involved in the regulation of their target genes and influences several cellular processes, including proliferation, differentiation, and apoptosis. Our previous studies have found that the miR-148/152 family was dysregulated in gastrointestinal cancers and was associated with biological characteristics of the tumor. Furthermore, miR-148/152 family members were regulated by single nucleotide polymorphisms and methylation of their CpG islands $[13,14]$. For example, miR-148b is differentially expressed in tumor and non-tumor tissues and is associated with the genesis and development of the disease [15]. We found that the expression of miR-148b was dysregulated in gastric cancer and colorectal cancer, and attempted to explain the mechanisms by which miR-148b regulates tumorigenesis and tumor progression in gastric and colorectal cancer [16, 17]. However, the mechanisms involved in the regulation of the miR-148/152 family in gastrointestinal cancers remain unclear.

LncRNAs are functional RNAs longer than 200 nucleotides and do not encode proteins. These molecules have a crucial role in several biological processes. LncRNAs can fold into complex secondary and higher-order structures to provide greater versatility and capability for protein and target recognition [18]. Improvements in high-resolution microarray analysis and genome sequencing technologies allowed the elucidation of lncRNAs. The dysregulated expression of $\operatorname{lncRNAs}$ is reported to be associated with several diseases, including many types of cancer [19]. Moreover, Salmena et al. [20] presented a unifying theory in which lncRNAs and microRNAs constitute competing endogenous RNAs (ceRNA), and played an important role in pathological conditions, such as cancers, and this theory greatly expanded our understanding of functional genetics.

The aim of the present study was to profile the expression of lncRNA that might be associated with miR-148b in gastric cancer and those that could improve the level of diagnosis and treatment of gastric cancer. Moreover, this study indicates that dysregulated lncRNAs may participate in the development of gastric cancer by interacting with miR-148b. These findings will elucidate the function of lncRNAs in gastric cancer and the effect of miR-148b on lncRNAs.

\section{Materials and methods}

Human gastric cancer cell culture. The human gastric cancer cell line SGC-7901 was purchased from the Type Culture Collection of the Chinese Academy of Sciences (Shanghai, China), and the human gastric cancer cell line AGS was purchased from The American Type Culture Collection (ATCC, USA). The SGC-7901 and AGS cell lines were cultured in RPMI 1640 medium (Hyclone) supplied with 10\% fetal bovine serum (Hyclone). The cell lines were cultured at $37^{\circ} \mathrm{C}$ and $5 \% \mathrm{CO}_{2}$ in a humidified atmosphere.

Cell transfection and of total RNA extraction. MiR-148b mimics were an RNA duplex purchased from GenePharma (Shanghai, China). The sequences of these mimics were described previously [16]. To improve RNA stability, all the pyrimidines of the mimics used in our study were substituted with 2-O-methyl analogues.

SGC-7901 cells transfected with miR-148b mimics were used as a test group and SGC-7901 cells without any treatment were used as a control group. Transfection was performed with Lipofectamine 2000 Reagent (Invitrogen) following the manufacturer's protocol. The final concentration of the miR$148 \mathrm{~b}$ mimics was $50 \mathrm{nM}$ in $2 \mathrm{ml}$ RPMI 1640 medium. After 48 hours of transfection, total RNA of cell samples was isolated using the TRIzol Reagent (Ambion) according to the manufacturer's protocol. The concentration and purity of total RNA were detected using a Nano-Photometer spectrophotometer in the UV and visible spectra (Implen), respectively. Real time RT-PCR was used to monitor the efficiency of transfection. All transfections were performed triplicate independently. All RNA samples were stored at $-80^{\circ} \mathrm{C}$ until further processing.

Microarray analysis. The expression of lncRNAs was profiled using the Agilent LncRNA + mRNA Human Gene Expression Microarray V3.0 platform which was designed for the profiling of human lncRNAs and protein-coding transcripts (CapitalBio, Beijing, China). The array contained 37581 lncRNAs and 34303 protein-coding transcripts. All lncRNAs included in the array were correctly chosen from robust databases such as ENSEMBL, GENCODE, RefSeq, UCSC, and other related resources. A splice junction probe or specific exon, which is used to identify individual transcripts accurately, was supplied for each transcript. Positive probes for house-keeping genes and negative probes were also printed onto the array for quality control of hybridization. We processed three samples for each treatment, for a total six samples. We prepared the samples using a cRNA amplification and labeling kit (CapitalbBio). The samples were reverse transcribed using CbcScript II reverse transcriptase and then labeled. After that, cDNA was generated by reverse transcription of total RNA obtained from cell samples using a reverse transcription kit (CapitalbBio) and hybridized using a CapitalBio BioMixer TM II Hybridization Station. After washing, slides were scanned using an Agilent DNA microarray scanner (G2565CA). The Agilent Feature Extraction software version 10.7 was used to analyze and extract the data on hybridization. 
Normalization and analysis of data were performed using the GeneSpring GX software version 13.0.

Real-time reverse-transcription PCR. Total RNA was isolated using Trizol reagent (Invitrogen) and reverse transcribed using PrimeScript RT Master Mix (Takara). Real-time reverse-transcription PCR (RT-PCR) was performed using the SYBR Premix Ex TaqTMII Kit (Takara) according to the manufacturer's instructions. The reactions were performed in a LightCycler 480II Real-Time PCR System (Roche) for 40 cycles at $95^{\circ} \mathrm{C}$ for $10 \mathrm{~s}, 60^{\circ} \mathrm{C}$ for 30 s. The primers used for real-time RT-PCR were designed and synthesized by Sangon Biotech (Shanghai, China). The primer sequences used were: M18204.1, forward: 5' GAGGAAGAAGGGACACTCCA 3', reverse: 5' TCGGTGAATAGGCAGACAGA 3' ; ENST00000548900.1, forward: 5' TGGGTTATTTCTTTGATAAGAACTG 3' , reverse: 5' GGCTCACTGCAACCTCCACCTC 3'; XLOC_006324, forward: 5' AGGAAGACGGAGCAGCACA 3', reverse: 5' TGTAATCCCAGCACTTTGG 3'; ENST00000420902.1, forward: 5' TTACCTCCCATCCATCATCC 3', reverse: 5' CAAGAAGCATAGCCACACAGG 3'; AK027145, forward: 5' TGCTGGCTGAATCCTTTCTT 3', reverse:5' GATGGTATGGCACTTTGGTG 3'; HIX0023999, forward: 5' CAGGGTGTTGTAAGCAGTGG 3', reverse: 5' GGTTGGGTGGTAGTCAGAGC 3'; XLOC_000983, forward: 5' AAGGAAGACGGAGCAGCA 3', reverse: 5' TGTAATCCCAGCACTTTGG 3'; P22857, forward: 5' GGATTTATTTCTGTGCCTGGA 3', reverse: 5' CCACCACTGTCTGCCTTCTT 3'. GAPDH was used as an internal control. Each sample was assayed in triplicate. The expression levels were determined by the $2^{-\Delta \Delta C T}$ method.

Gene Ontology (GO) and pathway analyses. The GO project (http://geneontology.org/) provides a controlled vocabulary for describing genes and gene products using three structured networks. The $\mathrm{p}$ value indicates the significance of the GO term enrichment of different mRNAs. P values of less than 0.05 were considered statistically significant. Pathway analysis was also performed for differentially expressed mRNAs using the KEGG database; this analysis was used to determine the biological pathways in KEGG of differentially expressed mRNAs. The $\mathrm{p}$ value indicated the significance of the pathway correlated with the conditions. Lower $\mathrm{p}$ values indicated higher significance.

Coding-non-coding gene co-expression network. The co-expression network of coding-non-coding genes (CNC network) was constructed for the differentially expressed lncRNAs and their targeted coding genes. Pearson correlation coefficient was calculated for each pair of genes and coefficients equal to or greater than 0.995 were chosen to draw the network using Cytoscape. In the network analysis, a degree was defined as the number of directly linked neighbors. The degree was considered the most important measure of the centrality of a gene within a network and indicated its relative importance.

Statistical analysis. The data were analyzed using SPSS software version 19.0 (SPSS, Chicago, IL, USA). All assays were repeated at least three times. The expression levels of mRNAs and lncRNAs were compared between two groups using Student's t-test. GO and pathway analyses were performed using Fisher's exact test. All quantitative data were expressed as mean \pm standard deviation (SD). A p-value of less than 0.05 was considered statistically significant.

\section{Results}

Differentially expressed lncRNAs. The results of the high throughput lncRNA microarray data indicated that 37581 lncRNAs were expressed in the gastric cancer cell line SGC7901 and in cell line SGC-7901 treated with miR-148b mimics. We used hierarchical clustering analysis to arrange cell lines into groups according to the expression levels, which could indicate distinct expression modes between these cell lines (Figure 1). Scatter plots were used to determine the expression levels of lncRNAs between these cell lines. We found that 304 lncRNAs (log fold-change $\geq 2$ ) were differentially expressed in these two cell lines. Among the differentially expressed lncRNAs, 240 were up-regulated and 64 were down-regulated lncRNA. The top ten differentially expressed lncRNAs in the up-regulated and down-regulated groups are shown in Table 1. XLOC_000983 (fold change: 25.3583) was the most up-regulated lncRNA, and M18204.1 (fold change: 33.9060) was the most down-regulated lncRNA. Furthermore, upregulated $\ln c \mathrm{RNAs}$ were more common than down-regulated lncRNAs in our analysis.

Differentially expressed mRNAs. The microarray results allowed the identification of 34303 mRNAs in the SGC-7901 cell lines. The mRNA expression modes, which were present in the SGC-7901 line treated with miR-148b mimics and the normal SGC-7901 cell line, were analyzed using the hierarchical clustering analysis. Among the differentially expressed mRNAs, 196 were consistently up-regulated, and 320 were consistently down-regulated. POSTN (fold change: 26.9686) was the most up-regulated mRNA, and HBB (fold change: 64.0279) was the most down-regulated mRNA.

Real-time PCR analysis. To confirm the microarray results, we randomly selected two up-regulated lncRNAs (XLOC_000983, XLOC_005104) and six down-regulated lncRNAs (M18204.1, ENST00000548900.1, XLOC_006324, ENST00000420902.1, AK027145, HIX0023999) with log foldchanges $>2$ to perform real-time PCR. The results of real-time PCR indicated a consistency of $87.5 \%$ between the real-time PCR results and the microarray results in SGC-7901 cells, these also showed a same result between PCR and microarray in AGS cells (Figure 2).

GO analysis. We performed the Gene Ontology (GO) analysis to caculate the enrichment of genes and gene products involved in molecular functions, cellular components, and biological processes. We used Fisher's exact test to determine the presence of overlaps between GO annotation list and the list of differentially expressed mRNAs. The $\mathrm{p}$ value indicates the significance of GO annotation enrichment in the differ- 
A

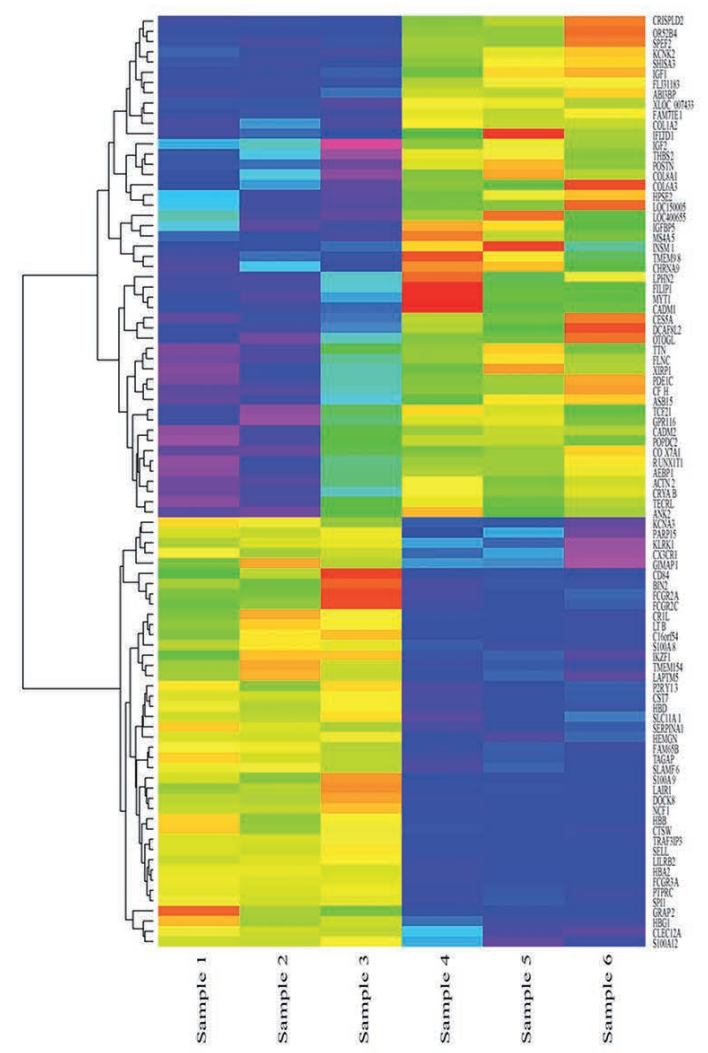

C

\section{Log-log scatter plot}

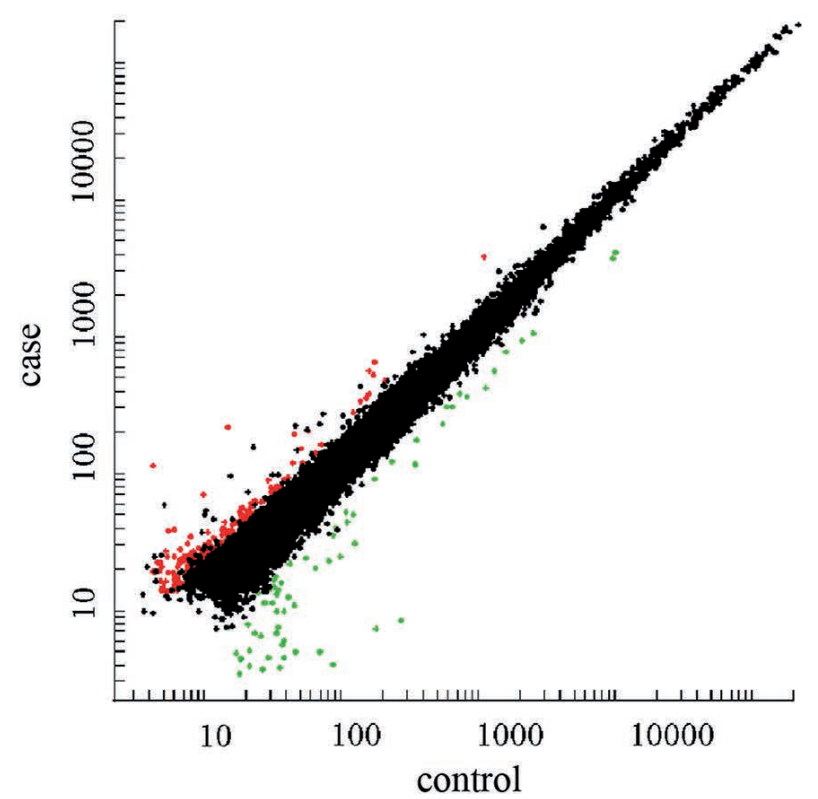

B

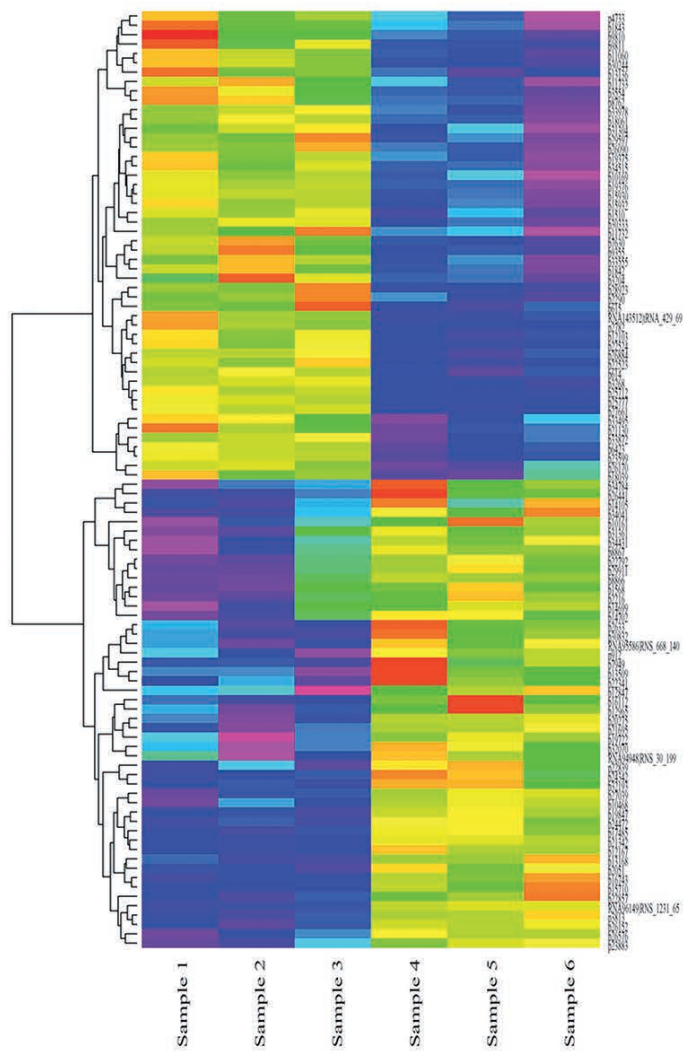

D

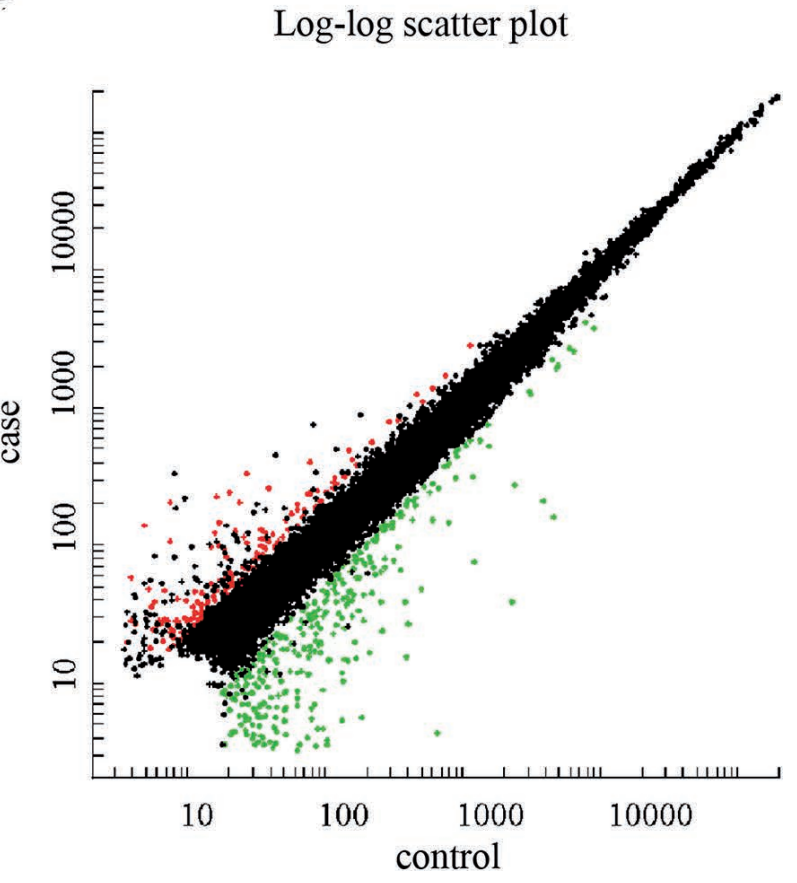

Figure 1. Microarray analysis of miR-148b-related lncRNA of gastric cancer lines. A. Heat map and hierarchical clustering dendrogram of lncRNA chips. B. Heat map and hierarchical clustering dendrogram of mRNA chips (greater than 2.0-fold; $P<0.05)$. In (A) and (B), samples 1-3 correspond to gastric cancer cells treated with miR-148b mimics, and samples 4-6 correspond to negative control cells. C. Scatter plot of the lncRNA expression profile. D. Scatter plot of the mRNA expression profile. In (C) and (D), samples in the case group correspond to gastric cancer cells treated with miR$148 \mathrm{~b}$ mimics, and samples in the control group correspond to negative control cells. 
Table 1. Top 10 differentially expressed lncRNAs.

\begin{tabular}{|c|c|c|c|c|c|c|c|}
\hline ProbeName & p-value & Regulation & Probe Sequence & LncRNA ID & Chromosome & Class & Database \\
\hline p3268 & 26.30557 & down & $\begin{array}{l}\text { CATCAGATGCCTTCATCAGCTGGTATTTT- } \\
\text { GCCTAAGATCTATTTAAGATAACCTTTTCTT }\end{array}$ & ENST00000548900.1 & 12 & Antisense & ENSEMBL \\
\hline p11060 & 15.19172 & down & $\begin{array}{l}\text { GTCATCATGTAGCTAGCAATGGCCACATCAAA- } \\
\text { GATACTATGAACAATTTTAAACAACACC }\end{array}$ & ENST00000420902.1 & 22 & Intergenic & ENSEMBL \\
\hline p1510 & 7.019455 & down & $\begin{array}{l}\text { CGCTGTACTGTTTTTTAGCCCTTTGACTCAT- } \\
\text { TAATGTATCAGGGAATAAAAAGGATATCA }\end{array}$ & ENST00000379256.3 & 10 & Intronic & ENSEMBL \\
\hline p15930 & 5.646037 & down & $\begin{array}{l}\text { GCTTTTGCAAGCTTCAGGAAAACCTTTACT- } \\
\text { CACAGGTGAGTTCCACCTGAGGTTAATTTG }\end{array}$ & ENST00000522865.1 & 8 & Intergenic & ENSEMBL \\
\hline p9355 & 4.531518 & down & $\begin{array}{l}\text { AACTCTCTTACAAACTTTGAGCCCTGAG- } \\
\text { CAAATCCTGTGGGATAATGTGCTTCTGCACGT }\end{array}$ & ENST00000442794.1 & 2 & Antisense & ENSEMBL \\
\hline p3554 & 4.482015 & down & $\begin{array}{l}\text { AGGGAGGAATTTTCAGATTGACCAAAGAG- } \\
\text { TATTGGTCTACATCATTAGTACTTTCAGTGA }\end{array}$ & ENST00000544591.1 & 12 & Intergenic & ENSEMBL \\
\hline p11733 & 4.449931 & down & $\begin{array}{l}\text { GATGAGACAGATGCTTTCTAAGTCCGA- } \\
\text { TATATCTACCCACTTTCTGCTGTCACCTCTGTA }\end{array}$ & ENST00000598149.1 & 3 & Intergenic & ENSEMBL \\
\hline p7630 & 4.287467 & down & $\begin{array}{l}\text { AAGGGGCTCAAAACCTAGACTTTATAAAACT- } \\
\text { GACTTTTCTTTGTCACTTTTCGGTGTTTA }\end{array}$ & ENST00000561598.1 & 18 & Intergenic & ENSEMBL \\
\hline p1842 & 4.271108 & down & $\begin{array}{l}\text { AACTAGTCAGTCCAAGTATTTCTAAAGATC- } \\
\text { CACAGACACAGTTCTCCTGGATTTGGGAAG }\end{array}$ & ENST00000414894.1 & 10 & Intergenic & ENSEMBL \\
\hline p15932 & 4.12965 & down & $\begin{array}{l}\text { CAAATGCAAGGATAATCTTACACGAGTCCAG- } \\
\text { GAGGAAGGCTCATTCCACACTAAGTGTTC }\end{array}$ & ENST00000517869.1 & 8 & Intergenic & ENSEMBL \\
\hline p1568 & 6.546019 & up & $\begin{array}{l}\text { ACATTTGTGGAAGACGGAGTGTGTTTCCAT- } \\
\text { TATCCTTTCAGAGTTTGCAAACGAGGCGGT }\end{array}$ & ENST00000456722.1 & 10 & Intergenic & ENSEMBL \\
\hline p16743 & 6.528198 & up & $\begin{array}{l}\text { TAAACACATACCATGAGAAAGGGATTCCAT- } \\
\text { GTCTTCCAATCCTGCCCAGAGATGCAGAGA }\end{array}$ & ENST00000436360.1 & 9 & Antisense & ENSEMBL \\
\hline p7949 & 6.031635 & up & $\begin{array}{l}\text { ACTTTTCCAGGATCCTGGAATTTGTTCTGTT- } \\
\text { GCTTTAACTTGCTTTCCTGTGGCTGAGCT }\end{array}$ & ENST00000584547.1 & 18 & Intergenic & ENSEMBL \\
\hline p2216 & 4.872983 & up & $\begin{array}{l}\text { TCCCTTTAAAGAAAGCTACGTAGAAGCAG- } \\
\text { GATGAGTAAAAATTTACAAACTGAAACAGGA }\end{array}$ & ENST00000526935.1 & 11 & Antisense & ENSEMBL \\
\hline p8866 & 4.641507 & up & $\begin{array}{l}\text { ATTCCCGGATAAGGAAGAAGAGAGTGTCAC- } \\
\text { CTACTTCAGTGTGGTTTCAACCCTACTTCT }\end{array}$ & ENST00000594783.1 & 19 & Divergent & ENSEMBL \\
\hline p8867 & 4.307474 & up & $\begin{array}{l}\text { TTCAGTGTGGTTTCAACCCTACTTCTGCATCT- } \\
\text { TAAAGACACTGTGTACAACGTTGGACAC }\end{array}$ & ENST00000299997.4 & 19 & Divergent & ENSEMBL \\
\hline p12847 & 3.962276 & up & $\begin{array}{l}\text { AGCACACACTTAAAGCACACAACTT- } \\
\text { GCTAAGTTGTTGAAATTCTCAACACAATCAA- } \\
\text { GATT }\end{array}$ & ENST00000437514.1 & 4 & Intergenic & ENSEMBL \\
\hline p13168 & 3.852412 & up & $\begin{array}{l}\text { GCCAAAGCTTTCTGTAATTGATTTTTCCTGCT- } \\
\text { TCTTTACCCATTTGTACCATTATGAAGA }\end{array}$ & ENST00000601500.1 & 5 & Intergenic & ENSEMBL \\
\hline p3813 & 3.815256 & up & $\begin{array}{l}\text { TCAGTGACCCAAAAAGGAACTAAACGGTAC- } \\
\text { CCAGACTAGAAATCAATGTCAAATTATTCA }\end{array}$ & ENST00000552584.1 & 12 & Intergenic & ENSEMBL \\
\hline p13509 & 3.309407 & up & $\begin{array}{l}\text { GTGTCAGAAAGTTAACAACAGCTAACTGTTT- } \\
\text { GTTGTGGTGTTCTGAGTAACTTGTATTAT }\end{array}$ & ENST00000506431.2 & 5 & Intergenic & ENSEMBL \\
\hline
\end{tabular}

entially expressed mRNAs. Smaller $p$ value indicated a higher significance of the GO terms $(\mathrm{p}<0.05)$. The results indicated that the highest enriched GOs targeted by up-regulated transcripts were metabolic processes involving L-arabinose (GO: 0046373; ontology: biological process; $p=2.02 \mathrm{E}-09$ ), extracellular matrix (GO: 0031012; ontology: cellular component; $\mathrm{p}=7.37 \mathrm{E}-07$ ), and alpha-N-arabinofuranosidase activity (GO: 0046556; molecular function; $\mathrm{p}=1.6 \mathrm{E}-09$ ). The highest enriched GOs targeted by the down-regulated transcripts were cell periphery (GO: 0071944; ontology: cellular component; $\mathrm{p}=3.47 \mathrm{E}-10)$ and antigen binding (GO: 0003823; molecular function; $\mathrm{p}=4.17 \mathrm{E}-13)$. In the biological process domain, the highest enriched GOs targeted by the down-regulated transcripts were associated with the immune system, including immune system processes (GO: 0002376), regulation of immune system processes (GO: 0002682), and immune response-regulating signaling pathways (GO: 0002764) (Supplemental Figure 1).

Pathway analysis. We performed pathway analysis, which is a functional analysis, to map genes to KEGG pathways. In the present study, four pathways corresponded to up-regulated transcripts and the most enriched network was "ECM-receptor interaction" (Fisher's p-value of 9.10E-07), composed of five targeted genes, and 33 pathways corresponded to 
A

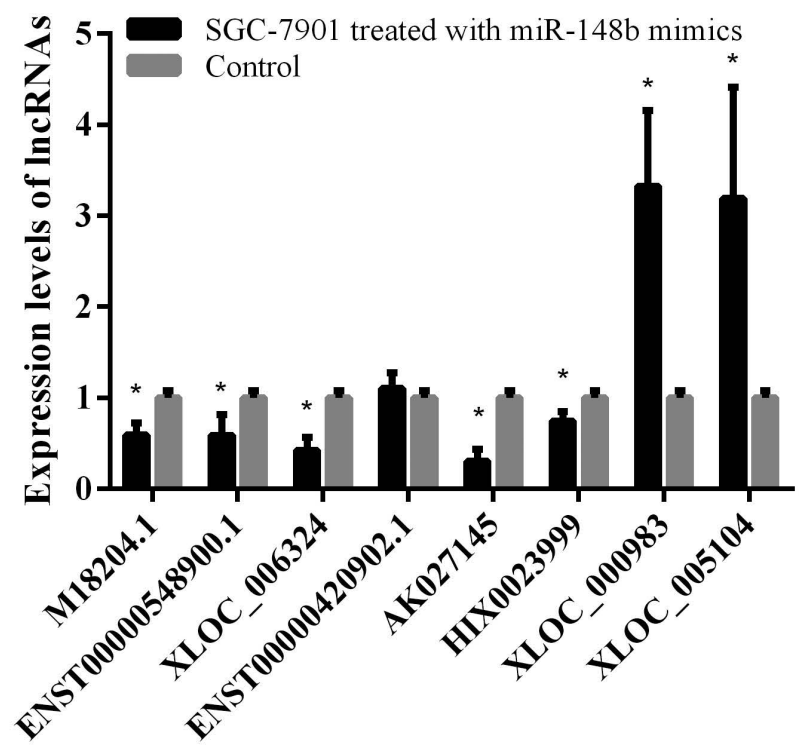

B

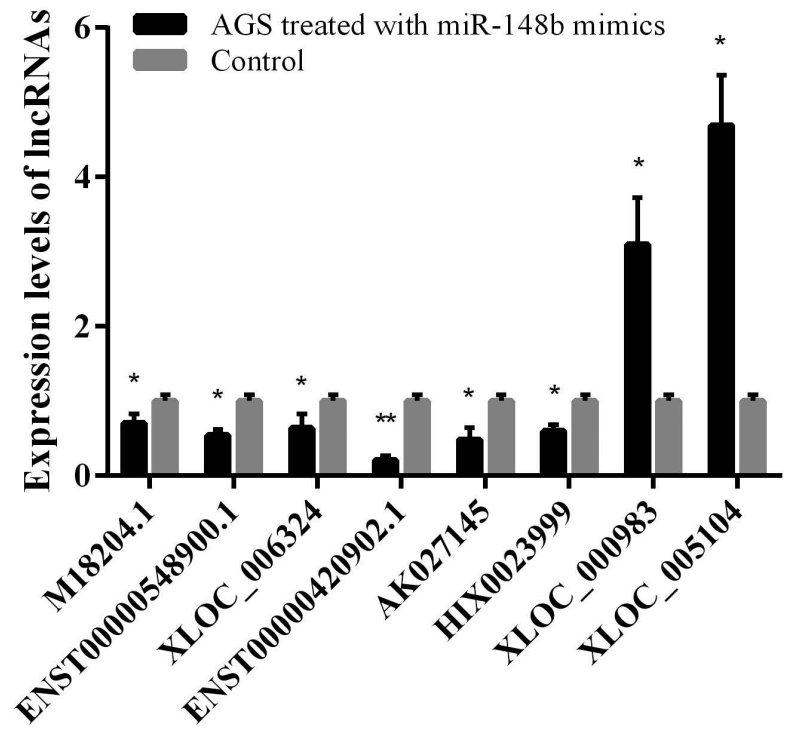

Figure 2. Differential expression of lncRNAs in gastric cancer cells treated with miR-148b mimics and normal gastric cancer cells by real-time PCR. A. Differential expression of IncRNAs in SGC-7901 cells. B. Differential expression of IncRNAs in AGS cells. ${ }^{\star} \mathrm{p}<0.05 ;{ }^{\star *} \mathrm{p}<0.01$.

down-regulated transcripts, and the most enriched network was "Staphylococcus aureus infection" (Fisher's p-value of 3.89E-15) composed of 23 targeted genes. Among the above pathways, the enriched up-regulated lncRNAs present in the category "focal adhesion" have been associated with gastric cancer (21). Furthermore, most pathways of the enriched down-regulated lncRNAs were associated with the immune system, including $\mathrm{T}$ cell receptor signaling pathway, intestinal immune network for IgA production, autoimmune thyroid disease, primary immunodeficiency, systemic lupus erythematosus, and rheumatoid arthritis. These results indicate that the lncRNAs that are influenced by miR-148b may play an important role in the immune system and are involved in the development of gastric cancer (Figure 3).

Co-expression network. Microarray-based CNC networks were constructed to cluster mRNAs and lncRNAs into a phenotypically correlated co-expression model. These networks were constructed on the basis of the correlation between differentially expressed mRNAs and lncRNAs in the gastric cancer cell lines SGC-7901 with and without treatment with miR-148b mimics. In these networks, the node included 272 lncRNAs and 463 mRNAs were positive. The network indicated that one lncRNA could correlate with more than one mRNA and one mRNA could correlate with more than one lncRNA. These results also indicated the potential internal adjustment correlation between the mRNAs and lncRNAs that are influenced by miR-148b in gastric cancer (Figure 4).

Bioinformatics analysis. Our results indicated that upregulated lncRNAs may influence the development of gastric cancer, and down-regulated lncRNAs may be associated with immune regulation. Therefore, we chose up-regulated lncRNAs which log fold-change $>2$ in treated and untreated gastric cancer cells and their associated genes with a function in focal adhesion. Moreover, we chose down-regulated lncRNAs which log fold-change $>2$ in treated and untreated gastric cancer cells and their associated genes with a function in immune regulation. We found that the up-regulated lncRNA RP11-99J16_A.2 was a natural antisense lncRNA located upstream of the CAPN9 gene. Previous studies reported that CAPN9 was associated with the prognosis of breast and colorectal cancer [22, 23]. However, the importance of RP11-99J16_A.2 in gastric cancer needs to be determined. In addition, we found that a down-regulated lncRNA RP111143G9.4, which was located downstream of LYZ gene. LYZ is an important gene in the immunosuppressive PD-1/PDL1 pathway and is involved in antigen presentation process [24]. Several studies have reported that LYZ might play an important role in breast cancer, colorectal cancer, and gastric cancer [25-27].

\section{Discussion}

Gastric cancer is one of the most common cancers and the second leading cause of cancer death in China [28]. The molecular mechanisms involved in gastric cancer have not been fully elucidated. Several studies have highlighted the importance of non-coding segments of the human genome in tumorigenesis and tumor progression. Among the noncoding RNAs, the role of miRNAs in gastric cancer has been elucidated. Our previous studies indicated that miR-148b plays a major role in the development of gastric cancer via regulation of CCKBR [16]. However, other potential targets of 
A

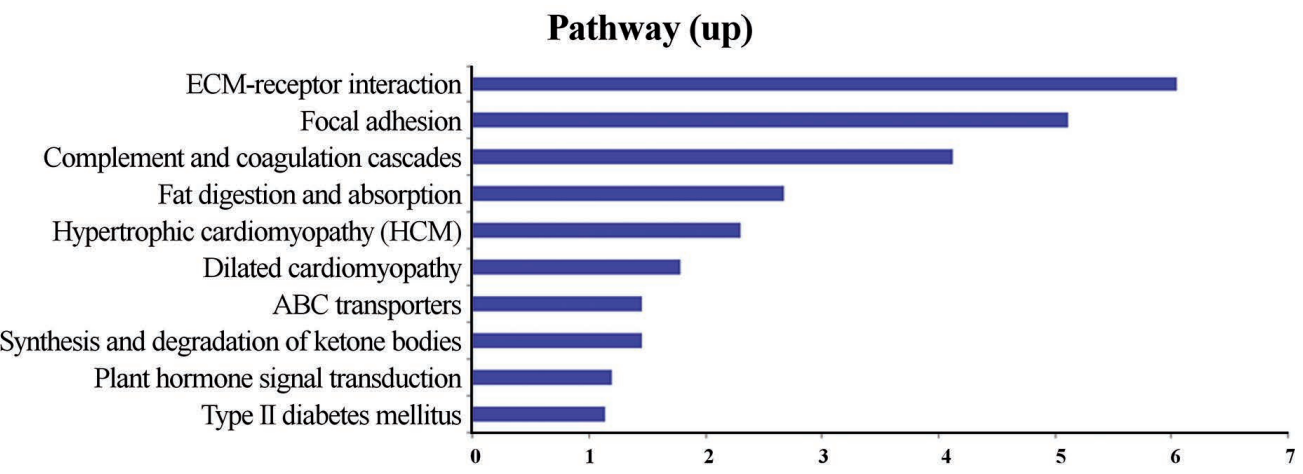

B

Pathway (down)

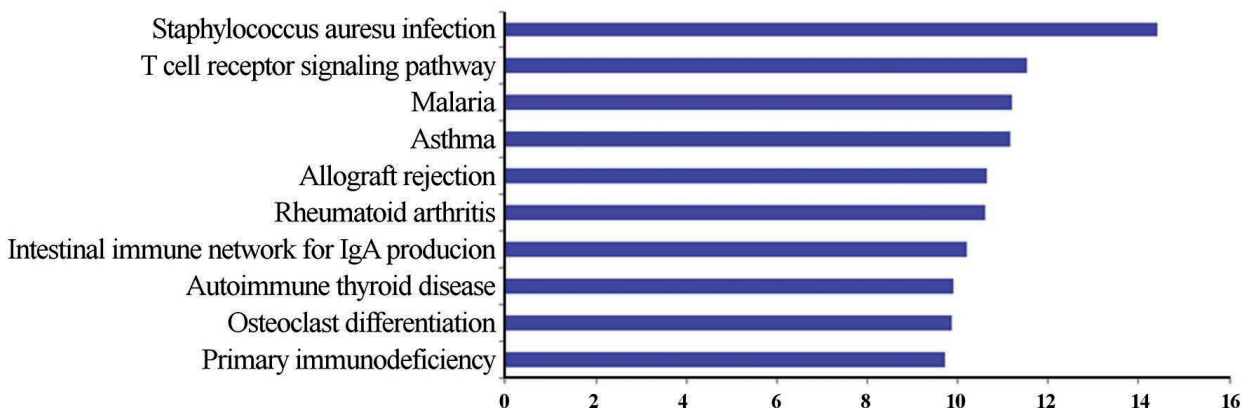

Figure 3. Pathway analysis. The top ten pathways of coding genes associated with up-regulated (A) and down-regulated (B) lncRNAs are listed.

miR-148b and its role in gastric cancer needed to be clarified. Furthermore, several studies have indicated that the dysregulated lncRNAs might be associated with tumorigenesis, as well as with development, metastasis, and prognosis of gastric cancer [29]. For example, the lncRNA H19, located on human chromosome 11, is dysregulated in gastric cancer [30]. H19 may stimulate cell proliferation, apoptosis, and metastasis as an oncogenic RNA in gastric cancer via regulation of p53, miR-675, and miR-141 [31-33].

Although the research on IncRNAs has increased quickly, only a few lncRNAs have been well studied. The functions and mechanisms of lncRNAs in gastric cancer are not fully understood. Along with this research, many theories have been proposed to explain the mechanisms of action of lncRNAs, including ceRNA hypothesis [20]. MiRNAs and lncRNAs are a critical part of ceRNAs and are involved in several biological processes, including the onset and development of tumors. The present study focused on the influence of miR-148b on lncRNAs and mRNAs in gastric cancer. The results of the microarray analysis indicated that the expression levels of $\ln c$ RNAs were altered compared with the untreated gastric cancer cells. Subsequently, we validated the microarray results using real-time PCR and the results corroborated the microarray results. Furthermore, the GO analysis revealed that the enriched GOs targeted by downregulated transcripts were associated with the immune system. Similarly, the pathway analysis indicated that most pathways of enriched down-regulated lncRNAs were related to the immune system. Then, we randomly chose the downregulated lncRNA RP11-1143G9.4, located downstream of the LYZ gene, to perform bioinformatics analysis. We found that LYZ was an important gene in the immunosuppressive PD-1/PD-L1 pathway and was involved in antigen presentation, and RP11-1143G9.4 might participate in immune regulation as an antisense IncRNA of LYZ. These results indicate that $\operatorname{lncRNAs}$ controlled by miR-148b may be key regulators of the immune network and are related with the immune microenvironment of gastric cancer.

Although chemotherapy and radiotherapy may be effective in some cases to consolidate treatment and prolong survival of gastric cancer, more attention should be paid to accurate and effective therapeutic measures such as immunotherapy [34]. Recently, the Cancer Genome Atlas (TCGA) proposed the classification of gastric cancer into four subgroups: tumors positive for Epstein-Barr virus (EBV), microsatellite-unstable tumors (MSI), genomically stable tumors, and unstable chromosomal unstable tumors [35]. The EBV and MSI subgroups may be involved in the immune response to gastric cancer. Different mechanisms used to prevent immune escape have been characterized, and PD-1/PD-L1 inhibitors such as pembrolizumab, avelumab, durvalumab, and atezolizumab have been effective for treatment of gastric cancer [36]. In addition, ncRNAs including miRNAs and lncRNAs may play a key role in immune regulation. Song et al [37] found that 


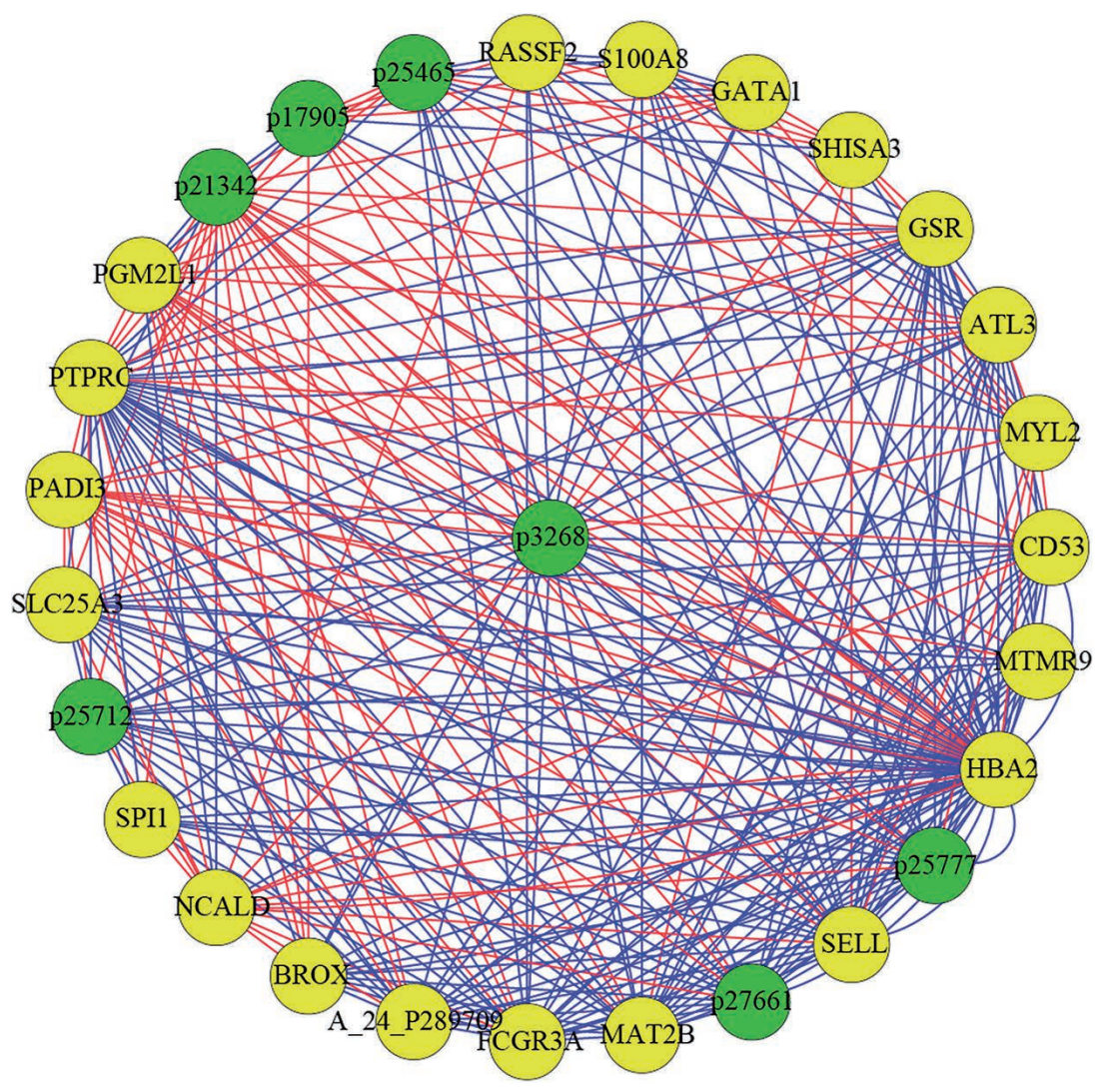

Figure 4. Coding-non-coding gene co-expression network between the differentially expressed lncRNAs and mRNAs in gastric cancer cell lines treated with miR-148b mimics and normal gastric cancer cells. Genes colored in yellow are protein-coding RNAs associated with gastric cancer. Genes colored in green are IncRNAs associated with gastric cancer.

the overexpression of lncRNA HOTAIR might be involved in tumor escape mechanisms, associated with the upregulation of HLA-G via inhibition of miR-152. Their results indicated the potential application of HOTAIR in gastric cancer immunotherapy for better prognosis and improved survival. Moreover, Liu et al [38] reported that the miR-148/152 family could act as fine-tuner in the regulation of the innate immune response and Ag-presenting capacity of dendritic cells by targeting CaMKIIa, leading to immune homeostasis and immune regulation. Our results indicated that miR-148b might regulate immune processes in gastric cancer by regulating the expression of lncRNAs, which were consistent with those of the genome-wide analysis of lncRNA expression [39]. However, the interactions between miR-148b and the immune system during gastric cancer progression are not well understood. To the best of our knowledge, our study is the first to report that miR-148b can regulate several lncRNAs and that these molecules are closely associated with the immune system and therefore can influence gastric cancer progression.

In conclusion, this study demonstrated that miR-148b was involved in the dysregulation of lncRNAs in gastric cancer cell lines. The $\operatorname{lncRNAs}$ regulated by miR-148b may participate in several biological processes, including the regulation of the immune system. Our results may help improve diagnosis, and identify therapeutic targets, and facilitate the development of novel, personalized treatment strategies. However, more studies are necessary to determine the molecular mechanisms and biological functions of lncRNAs regulated by miR-148b in gastric cancer.

Supplementary information is available in the online version of the paper.

Acknowledgements: This work was supported by National Science Foundation of China (81372550), Program of Education Department of Liaoning Province (L2014307), the Key Laboratory Program of Education Department of Liaoning Province (LZ2015076, LZ2015080)

\section{References}

[1] TORRE L A, BRAY F, SIEGEL R L, FERLAY J, LORTETTIEULENT J et al. Global cancer statistics, 2012. CA Cancer J Clin 2015; 65: 87-108. https://doi.org/10.3322/caac.21262 
[2] JEMAL A, SIEGEL R, WARD E, MURRAY T, XU J et al. Cancer statistics, 2006. CA Cancer J Clin 2006; 56: 106-130. https://doi.org/10.3322/canjclin.56.2.106

[3] SJOQUIST K M, ZALCBERG J R. Gastric cancer: past progress and present challenges. Gastric Cancer 2015; 18: 205-209. https://doi.org/10.1007/s10120-014-0437-0

[4] BANG Y J, VAN CUTSEM E, FEYEREISLOVA A, CHUNG $\mathrm{H}$ C, SHEN L et al. Trastuzumab in combination with chemotherapy versus chemotherapy alone for treatment of HER2-positive advanced gastric or gastro-oesophageal junction cancer (ToGA): a phase 3, open-label, randomised controlled trial. Lancet 2010; 376: 687-697. https://doi. org/10.1016/S0140-6736(10)61121-X

[5] LI J, QIN S, XU J, GUO W, XIONG J et al: Apatinib for chemotherapy-refractory advanced metastatic gastric cancer: results from a randomized, placebo-controlled, parallel-arm, phase II trial. J Clin Oncol 2013; 31: 3219-3225. https://doi. org/10.1200/JCO.2013.48.8585

[6] DENG G, SUI G. Noncoding RNA in oncogenesis: a new era of identifying key players. Int J Mol Sci 2013; 14: 18319-18349. https://doi.org/10.3390/ijms140918319

[7] BROWN JD, MITCHELL SE, O'NEILL RJ. Making a long story short: noncoding RNAs and chromosome change. Heredity (Edinb) 2012; 108: 42-49. https://doi.org/10.1038/ hdy.2011.104

[8] CHEN Q, GE X, ZHANG Y, XIA H, YUAN D et al. Plasma miR-122 and miR-192 as potential novel biomarkers for the early detection of distant metastasis of gastric cancer. Oncol Rep 2014; 31: 1863-1870.

[9] IINO I, KIKUCHI H, MIYAZAKI S, HIRAMATSU Y, OHTA $\mathrm{M}$ et al. Effect of miR-122 and its target gene cationic amino acid transporter 1 on colorectal liver metastasis. Cancer Sci 2013; 104: 624-630. https://doi.org/10.1111/cas.12122

[10] FORNARI F, GRAMANTIERI L, GIOVANNINI C, VERONESE A, FERRACIN $M$ et al. MiR-122/cyclin G1 interaction modulates p53 activity and affects doxorubicin sensitivity of human hepatocarcinoma cells. Cancer Res 2009; 69: 5761-5767. https://doi.org/10.1158/0008-5472.CAN-08$\underline{4797}$

[11] LANFORD RE, HILDEBRANDT-ERIKSEN ES, PETRI A, PERSSON R, LINDOW $\mathrm{M}$ et al. Therapeutic silencing of microRNA-122 in primates with chronic hepatitis C virus infection. Science 2010;327: 198-201. https://doi.org/10.1126/ science. 1178178

[12] CHEN Y, SONG Y, WANG Z, YUE Z, XU H et al. Altered expression of MiR-148a and MiR-152 in gastrointestinal cancers and its clinical significance. J Gastrointest Surg 2010; 14: 1170-1179. https://doi.org/10.1007/s11605-010-1202-2

[13] CHEN X, WANG G, LU X, GAO P, SONG Y et al. Polymorphisms and haplotypes of the miR-148/152 family are associated with the risk and clinicopathological features of gastric cancer in a Northern Chinese population. Mutagenesis 2014; 29: 401-407. https://doi.org/10.1093/mutage/geu050

[14] SUN J, SONG Y, WANG Z, WANG G, GAO P et al. Clinical significance of promoter region hypermethylation of microRNA-148a in gastrointestinal cancers. Onco Targets Ther 2014; 7: 853-863.
[15] CHEN Y, SONG YX, WANG ZN. The microRNA-148/152 family: multi-faceted players. Mol Cancer 2013; 12: 43. https:// doi.org/10.1186/1476-4598-12-43

[16] SONG YX, YUE ZY, WANG ZN, XU YY, LUO Y et al. MicroRNA-148b is frequently down-regulated in gastric cancer and acts as a tumor suppressor by inhibiting cell proliferation. Mol Cancer 2011; 10: 1. https://doi.org/10.1186/1476-4598-10-1

[17] SONG Y, XU Y, WANG Z, CHEN Y, YUE Z et al. MicroRNA148 b suppresses cell growth by targeting cholecystokinin-2 receptor in colorectal cancer. Int J Cancer 2012; 131: 10421051. https://doi.org/10.1002/ijc.26485

[18] GUTTMAN M, RINN JL. Modular regulatory principles of large non-coding RNAs. Nature 2012; 482: 339-346. https:// doi.org/10.1038/nature10887

[19] SPIZZO R, ALMEIDA MI, COLOMBATTI A, CALIN GA: Long non-coding RNAs and cancer: a new frontier of translational research? Oncogene 2012; 31:4577-4587. https://doi. org/10.1038/onc.2011.621

[20] SALMENA L, POLISENO L, TAY Y, KATS L, PANDOLFI PP. A ceRNA hypothesis: the Rosetta Stone of a hidden RNA language? Cell 2011; 146: 353-358. https://doi.org/10.1016/j. cell.2011.07.014

[21] DU T, QU Y, LI J, LI H, SU L et al. Maternal embryonic leucine zipper kinase enhances gastric cancer progression via the FAK/Paxillin pathway. Mol Cancer 2014; 13: 100. https://doi. org/10.1186/1476-4598-13-100

[22] DAVIS J, MARTIN SG, PATEL PM, GREEN AR, RAKHA EA et al. Low calpain-9 is associated with adverse disease-specific survival following endocrine therapy in breast cancer. BMC Cancer 2014; 14: 995. https://doi.org/10.1186/1471-2407-14$\underline{995}$

[23] VAISH V, SANYAL SN. Role of Sulindac and Celecoxib in chemoprevention of colorectal cancer via intrinsic pathway of apoptosis: exploring NHE-1, intracellular calcium homeostasis and Calpain 9. Biomed Pharmacother 2012; 66: 116-130. https://doi.org/10.1016/j.biopha.2011.11.019

[24] TAUBE JM, YOUNG GD, MCMILLER TL, CHEN S, SALAS JT et al. Differential Expression of Immune-Regulatory Genes Associated with PD-L1 Display in Melanoma: Implications for PD-1 Pathway Blockade. Clin Cancer Research 2015; 21: 3969-3976. https://doi.org/10.1158/1078-0432.CCR-15$\underline{0244}$

[25] MAHANTA S, PAUL S, SRIVASTAVA A, PASTOR A, KUNDU $B$ et al. Stable self-assembled nanostructured hen egg white lysozyme exhibits strong anti-proliferative activity against breast cancer cells. Colloids Surf B Biointerfaces 2015; 130: 237-245. https://doi.org/10.1016/j.colsurfb.2015.04.017

[26] YIN HR, ZHANG L, XIE LQ, HUANG LY, XU Y et al. Hyperplex-MRM: a hybrid multiple reaction monitoring method using mTRAQ/iTRAQ labeling for multiplex absolute quantification of human colorectal cancer biomarker. J Proteome Res 2013; 12: 3912-3919. https://doi.org/10.1021/ pr4005025

[27] PARK JH, PARK J, CHOI JK, LYU J, BAE MG et al. Identification of DNA methylation changes associated with human gastric cancer. BMC Med Genomics 2011; 4: 82. https://doi. org/10.1186/1755-8794-4-82 
[28] CHEN W, ZHENG R, BAADE PD, ZHANG S, ZENG H et al. Cancer statistics in China, 2015. CA Cancer J Clin 2016; 66: 115-132. https://doi.org/10.3322/caac. 21338

[29] WANG J, SUN J, WANG J, SONG Y, GAO P et al. Long noncoding RNAs in gastric cancer: functions and clinical applications. Onco Targets Ther 2016; 9: 681-697. https://doi. org/10.2147/OTT.S95412

[30] ZHANG EB, HAN L, YIN DD, KONG R, DE W et al. c-Mycinduced, long, noncoding $\mathrm{H} 19$ affects cell proliferation and predicts a poor prognosis in patients with gastric cancer. Med Oncol 2014; 31: 914. https://doi.org/10.1007/s12032-0140914-7

[31] YANG F, BI J, XUE X, ZHENG L, ZHI K et al. Up-regulated long non-coding RNA H19 contributes to proliferation of gastric cancer cells. FEBS J 2012; 279: 3159-3165. https://doi. org/10.1111/j.1742-4658.2012.08694.x

[32] CAI X, CULLEN BR. The imprinted H19 noncoding RNA is a primary microRNA precursor. RNA 2007; 13: 313-316. https://doi.org/10.1261/rna.351707

[33] ZHOU X, YE F, YIN C, ZHUANG Y, YUE G et al. The Interaction Between MiR-141 and lncRNA-H19 in Regulating Cell Proliferation and Migration in Gastric Cancer. Cell Physiol Biochem 2015; 36: 1440-1452. https://doi. org/10.1159/000430309

[34] GOERE D, GRAS-CHAPUT N, AUPERIN A, FLAMENT C, MARIETTE $\mathrm{C}$ et al. Treatment of gastric peritoneal carcino- matosis by combining complete surgical resection of lesions and intraperitoneal immunotherapy using catumaxomab. BMC Cancer 2014; 14: 148. https://doi.org/10.1186/14712407-14-148

[35] CANCER GENOME ATLAS RESEARCH NETWORK. Comprehensive molecular characterization of gastric adenocarcinoma. Nature 2014; 513: 202-209. https://doi. org/10.1038/nature 13480

[36] ALSINA M, MOEHLER M, HIERRO C, GUARDENO R, TABERNERO J: Immunotherapy for Gastric Cancer: A Focus on Immune Checkpoints. Target Oncol 2016; 4: 469-477. https://doi.org/10.1007/s11523-016-0421-1

[37] SONG B, GUAN Z, LIU F, SUN D, WANG K et al. Long non-coding RNA [HOTIR promotes HLA-G expression via inhibiting miR-152 in gastric cancer cells. Biochem Biophys Res Commun 2015; 464: 807-813. https://doi.org/10.1016/j. bbrc.2015.07.040

[38] LIU X, ZHAN Z, XU L, MA F, LI D et al. MicroRNA$148 / 152$ impair innate response and antigen presentation of TLR-triggered dendritic cells by targeting CaMKIIalpha. J Immunol 2010; 185: 7244-7251. https://doi.org/10.4049/ ¡immunol.1001573

[39] SHORE AN, HERSCHKOWITZ JI, ROSEN JM. Noncoding RNAs involved in mammary gland development and tumorigenesis: there's a long way to go. J Mammary Gland Biol Neoplasia 2012; 17: 43-58. https://doi.org/10.1007/s10911-012-9247-3 
A

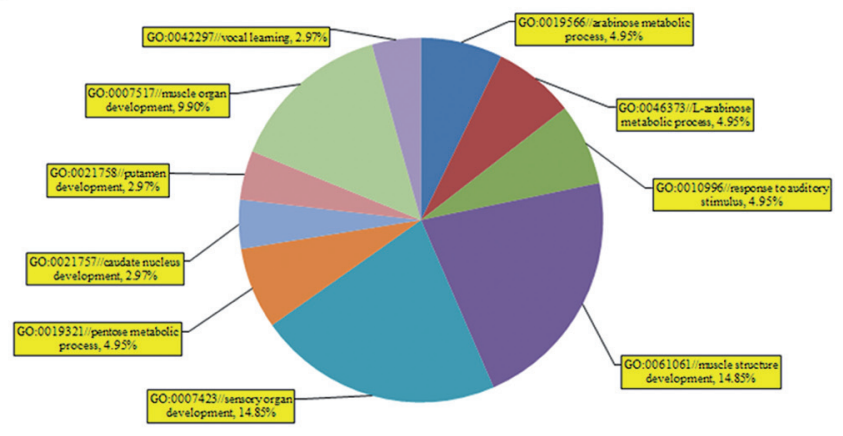

C

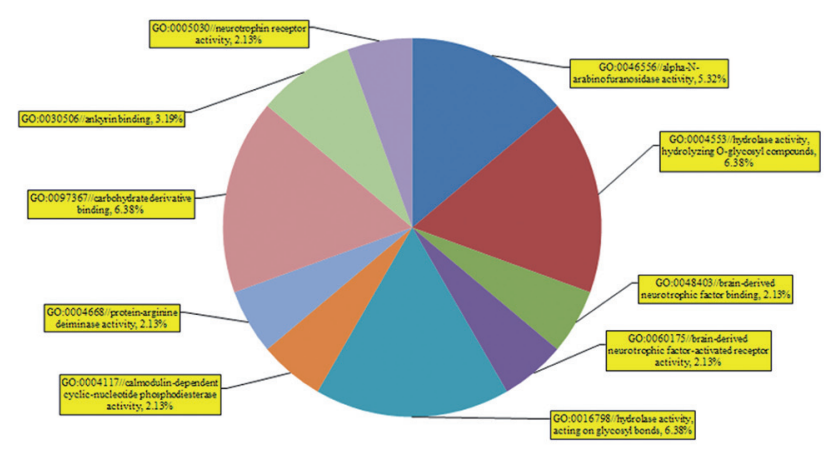

$\mathrm{E}$

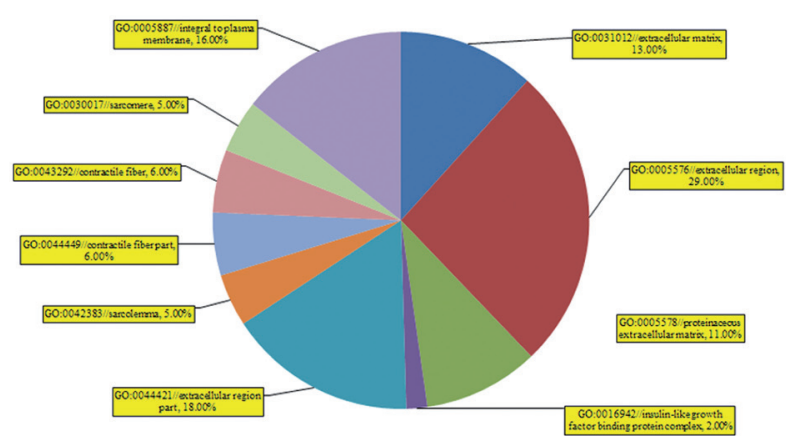

B

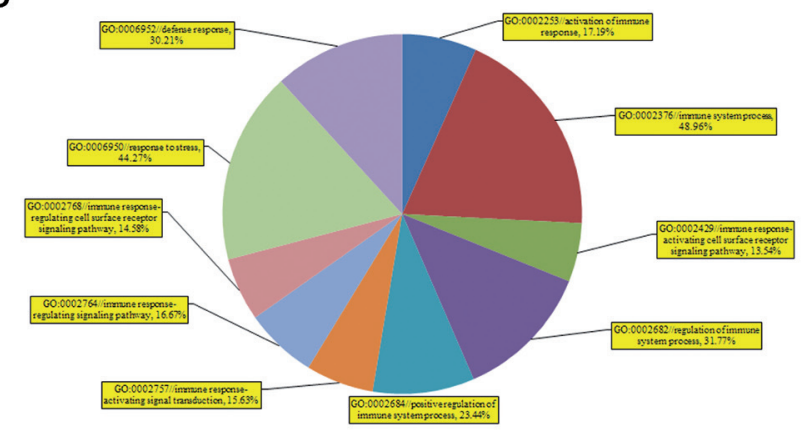

D

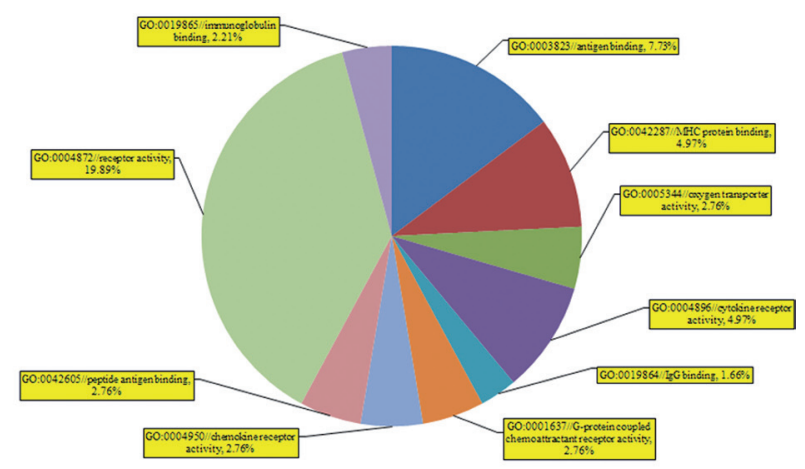

$\mathrm{F}$

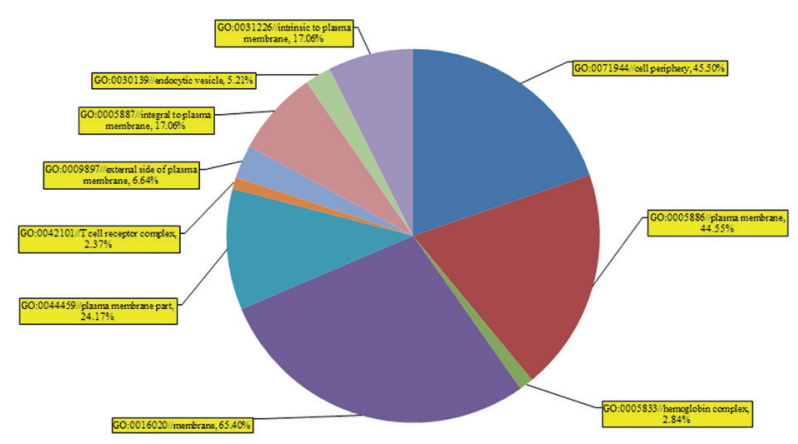

Supplemental Figure 1. Gene Ontology analysis. The top $10 \mathrm{GO}$ terms for the biological process of the coding genes associated with up-regulated (A) and down-regulated (B) IncRNAs are listed. The top $10 \mathrm{GO}$ terms for the molecular function of the coding genes associated with up-regulated (C) and down-regulated (D) IncRNAs are listed. The top $10 \mathrm{GO}$ terms for the cellular component of the coding genes associated with up-regulated (E) and down-regulated (F) lncRNAs are listed. 